\title{
Influence of temperature on AC conductivity of nanocrystalline $\mathrm{CuAlO}_{2}$
}

\author{
T Prakash $^{*}$
}

\begin{abstract}
Nanocrystalline $\mathrm{CuAlO}_{2}$ was synthesized by mechanical alloying of $\mathrm{Cu}_{2} \mathrm{O}$ and $\mathrm{a}-\mathrm{Al}_{2} \mathrm{O}_{3}$ powders in the molar ratio of 1:1 for $20 \mathrm{~h}$ in toluene medium with tungsten carbide balls and vials using planetary ball mill. The ball milling was carried out at $300 \mathrm{rpm}$ with a ball to powder weight ratio of 10:1 and then annealed at $1373 \mathrm{~K}$ in a platinum crucible for $20 \mathrm{~h}$ to get $\mathrm{CuAlO}_{2}$ phase with average crystallite size $45 \mathrm{~nm}$. Complex impedance spectroscopic measurement in the frequency region $1 \mathrm{~Hz}$ to $10 \mathrm{MHz}$ between the temperatures 333 to $473 \mathrm{~K}$ was carried out for nanocrystalline $\mathrm{CuAlO}_{2}$ sample. The obtained complex impedance data was analyzed for AC conductivities, DC and AC conductivities correlations and crossover frequencies $\left(f_{c o}\right)$. The BNN (Barton, Nakajima and Namikawa) relation was applied to understand the correlation between DC and AC conductivities. The observed experimental results were discussed in the paper.
\end{abstract}

Keywords: AC conductivity, Impedance spectroscopy, Transparent semiconductors

\section{Background}

Transparent electronic devices such as bipolar transistors and diodes can be fabricated with the help of $\mathrm{p}-\mathrm{n}$ transparent heterojunction [1]. The discovery of first transparent p-type semiconductor $\mathrm{CuAlO}_{2}$ in 1997 by Kawazoe et.al [2] triggered the development of 'transparent electronics' field. Reducing crystallite size of materials from micron to the nanodimension led to fundamental and technological importance due to their enhanced properties. Because if the crystallite size reduced to nanoscale the volume fraction of the atoms residing in the grain boundaries are increased so it induce dramatic changes in their electrical and dielectric properties which are important for their applications. The inter-granular regions often have a significant effect on the flow of electronic current. It gives rise to some very interesting and useful properties which are exploited in many commercial devices, but this depends on the method of material preparation and post processing treatments. Recently Gao et.al [3] observed the enhancement of electrical conductivity from $1 \mathrm{~S} / \mathrm{cm}$ to $2.4 \mathrm{~S} / \mathrm{cm}$ when the crystallite size was reduced to nanodimension. Techniques like pulsed laser deposition [2],

Correspondence: thanigaiprakash@gmail.com

Department of Medical Bionanotechnology, Chettinad Hospital and Research Institute, Kelambakkam, Tamilnadu 603103, India ion exchange reaction [3] and sputtering [4] were widely used to synthesis single phase $\mathrm{CuAlO}_{2}$. Recently the author reported the synthesis of nanocrystalline $\mathrm{CuAlO}_{2}$ by mechanical alloying process using $\mathrm{Cu}_{2} \mathrm{O}$ and $\alpha-\mathrm{Al}_{2} \mathrm{O}_{3}$ powders, the grain and grain boundary contribution electrical conductivities [5] and the existence of Debye type dielectric relaxation behaviour [6]. This paper reports the AC conductivity, scaling behaviour, correlations between DC and AC conductivities and cross-over frequencies of nanocrystalline $\mathrm{CuAlO}_{2}$.

\section{Experimental details}

The nanocrystalline $\mathrm{CuAlO}_{2}$ was synthesized by mechanical alloying of $\mathrm{Cu}_{2} \mathrm{O}$ and $\alpha-\mathrm{Al}_{2} \mathrm{O}_{3}$ powders in the molar ratio of 1:1 for $20 \mathrm{~h}$ using Fritsch Pulveristte-p5 planetary ball mill. The milling was carried out at $300 \mathrm{rpm}$ with a ball to powder weight ratio of 10:1 and then annealed at $1373 \mathrm{~K}$ in air atmosphere for $20 \mathrm{~h}$ to get $\mathrm{CuAlO}_{2}$ phase with average crystallite size of $45 \mathrm{~nm}$. The nanocrystalline $\mathrm{CuAlO}_{2}$ sample was made into cylindrical pellet of $5 \mathrm{~mm}$ diameter and $1 \mathrm{~mm}$ thickness at a pressure of 4 ton using a hydraulic press for impedance measurements. For good electrical contacts, silver paste has been coated on either flat sides of the pellets and then loaded in between two spring contact platinum electrodes. Impedance spectroscopic measurements were 
performed in the frequency range of $1 \mathrm{~Hz}$ to $10 \mathrm{MHz}$ with the applied potential of $50 \mathrm{mV}$ using Solartron $1260 \mathrm{im}$ pedance/gain phase analyzer which has been automated with a computer interface data acquisition system. The measurement was carried out in temperature range from 333 to 473 K. Shielded test leads were used for electrical connections from the analyzer to the sample in order to avoid any parasitic impedance. The temperature was measured by a platinum thermocouple located close to the sample. The temperature was controlled with an accuracy of $\pm 1 \mathrm{~K}$ by Eurotherm (818P) proportional integral differential (PID) temperature controller.

\section{Results and discussions}

Bode mode and phase plots are used to communicate frequency response of a material by complex impedance function $Z^{*}(\omega)=Z^{\prime}(\omega)-j Z^{\prime \prime}(\omega)$, where $Z^{\prime}$ $(\omega)=(|Z| \cos \theta)$ and $Z^{\prime \prime}(\omega)=(|Z| \sin \theta)$ are the real and imaginary parts of $Z^{*}(\omega), \omega(=2 \pi f)$ is the angular frequency, $f$ is the applied frequency, the phase angle $(\theta)$ is $\tan ^{1}\left[Z^{\prime \prime}(\omega) / Z^{\prime}(\omega)\right]$, and $|Z|=\left[Z^{\prime}(\omega) 2+Z^{\prime \prime}(\omega) 2\right] 1 / 2$. Bode mode and phase plots are used to represent the gain and phase of a system as a function of frequency. The magnitude plot is between magnitude and logarithmic frequency. This is useful to show the transfer

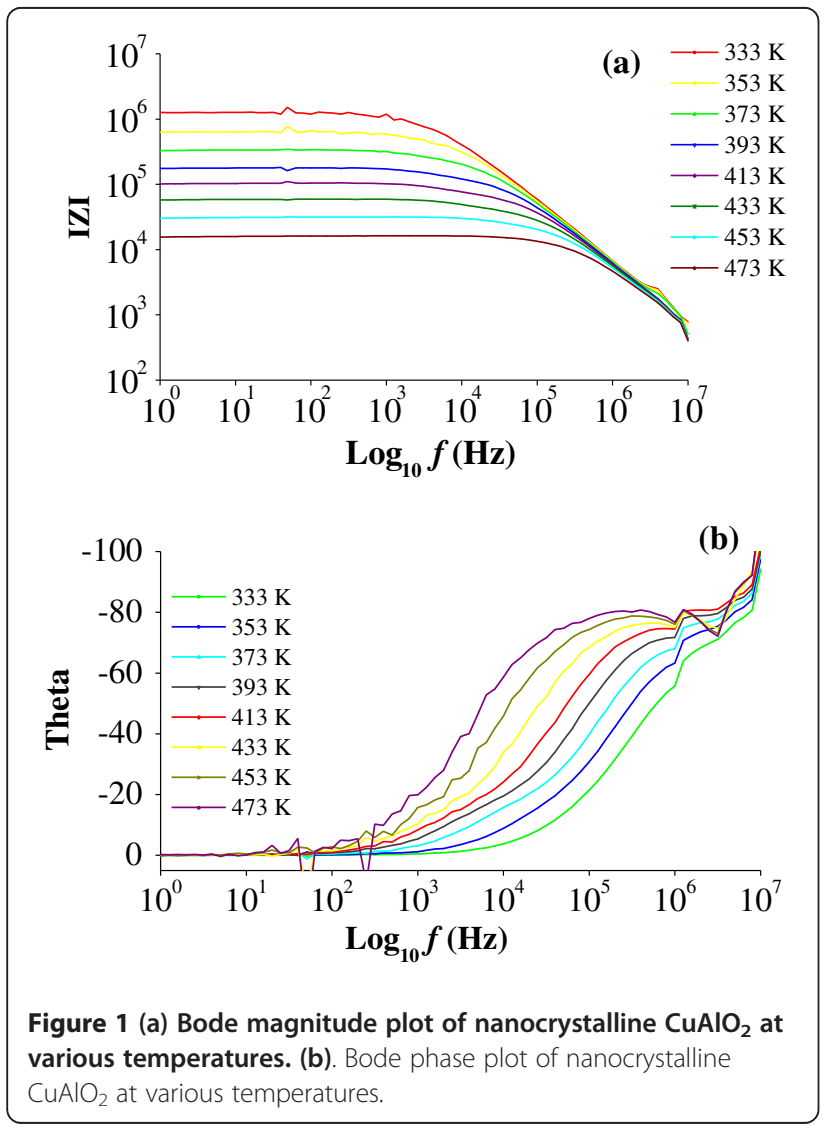

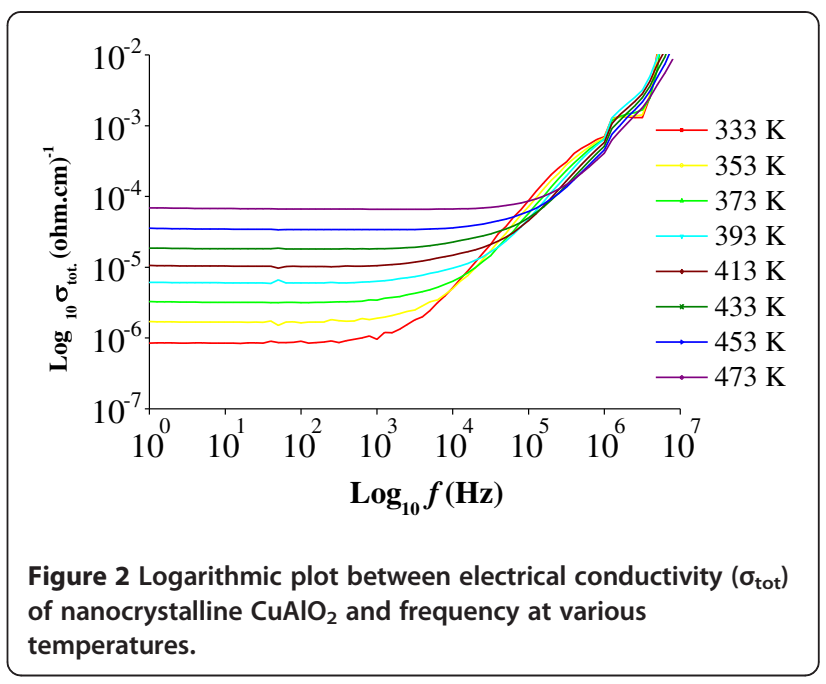

function or frequency response of a linear, timeinvariant system. The phase plot is between phase and logarithmic frequency, usually plotted in conjunction with the magnitude plot to evaluate how much a frequency will be shifted. Bode magnitude and phase plots of $\mathrm{CuAlO}_{2}$ at different applied temperatures are respectively shown in Figure 1(a) and (b). In both plots, different symbols represent the experimental data at different temperatures. The magnitude decreases with increase of temperature in the low frequency, but in higher frequency region it does not show any noticeable variation. In the phase plot, low frequency region data do not show any appreciable variation for all the measured temperatures.

The total electrical conductivity of nanocrystalline $\mathrm{CuAlO}_{2}$ at various temperatures was calculated using the following relation,

$$
\sigma_{t o t}=\frac{1}{\mathrm{Z}^{\prime}}\left(\frac{4 l}{\pi d^{2}}\right)
$$

where $Z^{\prime}(\omega)$ is the real part of $Z^{*}(\omega)$, / is the thickness

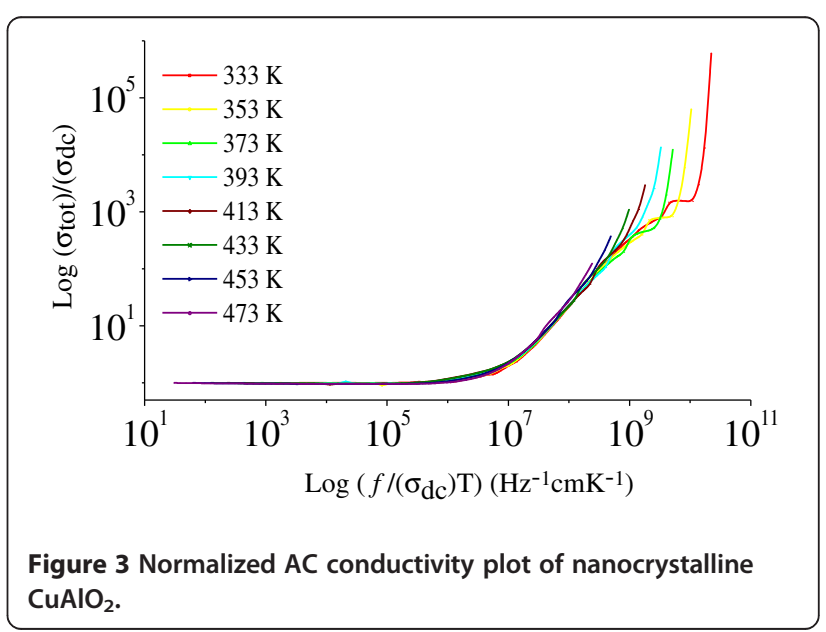




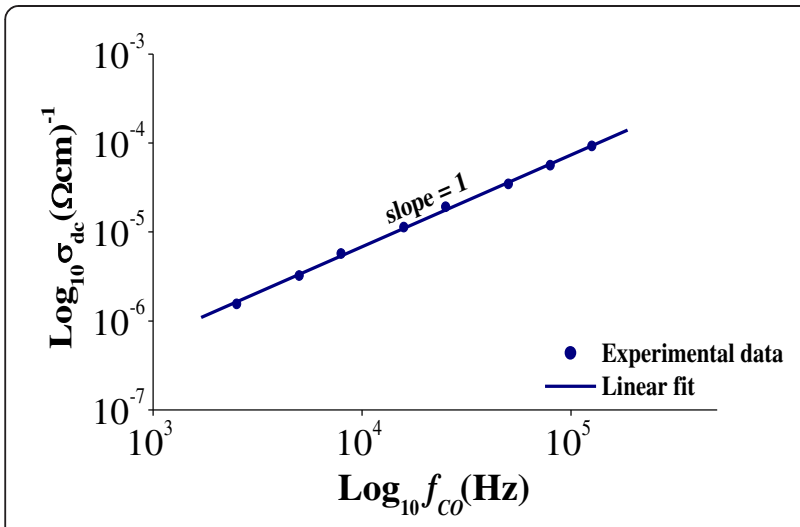

Figure 4 Logarithmic plot between DC conductivity $\left(\sigma_{\mathrm{dc}}\right)$ and cross over frequency $\left(f_{\mathrm{co}}\right)$, the solid line is the linear fit.

of the pellet and $d$ is the diameter of the pellet. Logarithmic plot between electrical conductivity $\left(\sigma_{\text {tot }}\right)$ of nanocrystalline $\mathrm{CuAlO}_{2}$ and frequency at various temperatures is shown in Figure 2. The conductivity is found to be frequency independent in the low frequency region and at high frequencies the conductivity dispersion has been observed. As the temperature increases, the frequency at which the dispersion becomes prominent shifts to higher frequency region. This total conductivity $\left(\sigma_{\mathrm{tot}}\right)$ is the summation of DC $\left(\sigma_{\mathrm{dc}}\right)$ and $\mathrm{AC}$ conductivities $\left(\sigma_{\mathrm{ac}}\right)$. So,

$$
\sigma_{t o t}=\sigma_{d c}+\sigma_{a c}(\omega)
$$

The DC conductivity $\left(\sigma_{\mathrm{dc}}\right)$ is independent of frequency and is due to free charge carriers in the system which was extracted from the extrapolation of frequency independent plateau region of the plot. In the conductivity normalized approach, the conductivity axis has been normalized with respect to $\sigma_{\mathrm{dc}}$ and the frequency axis with respect to $\sigma_{\mathrm{dc}} \mathrm{T}$, where $\mathrm{T}$ is the temperature in Kelvin. The normalized conductivity plot of nanocrystalline $\mathrm{CuAlO}_{2}$ is shown in Figure 3. All the conductivity curves collapse into a single master curve, which indicates that under conductivity formalism the relaxation mechanism is found to be temperature independent. The overlapping of conductivity curve into single master curve indicates the time-temperature superposition in the scaling. It is interesting to correlate the DC and AC conductivities in nanocrystalline materials. One of such correlation is the BNN relation developed by Barton, Nakajima and Namikawa [7]. The BNN relation is

$$
\sigma_{d c}=\left[\frac{1}{4 \pi^{\prime} H_{R}}\right] \epsilon_{O} \Delta \epsilon f_{C O}
$$

where $H_{R}$ is the Heaven ratio, $\epsilon_{0}$ is the permittivity of vacuum, $\Delta \epsilon=\left(\epsilon_{\mathrm{o}}-\epsilon_{\infty}\right)$ is the permittivity change from the unrelaxed baseline $\left(\epsilon_{\infty}\right)$ to the fully relaxed level $\left(\epsilon_{\mathrm{o}}\right)$ and $f_{\text {co }}$ is the cross over frequency [8]. The crossover frequency $\left(f_{c o}\right)$ is the frequency at which the conductivity independent turns to conductivity dependent. Logarithmic plot between DC conductivity $\left(\sigma_{\mathrm{dc}}\right)$ and cross over frequency $\left(f_{\text {co }}\right)$ is shown in Figure 4 . The solid line is a linear fit of the data which gives unity slope, implying that the $\mathrm{DC}$ and $\mathrm{AC}$ conductions are closely correlated to each other and that they are of the same mechanism.

\section{Conclusions}

Influence of temperature on AC conductivity behaviour and correlations between $\mathrm{DC}$ and $\mathrm{AC}$ conductivities using BNN relation for the nanocrystalline $\mathrm{CuAlO}_{2}$ were analyzed using the experimental data obtained from the impedance spectroscopic measurement in the frequency region $1 \mathrm{~Hz}$ to $10 \mathrm{MHz}$ between the temperatures 333 to $473 \mathrm{~K}$. The overlapping of conductivity curves into a single curve indicates the time-temperature superposition in the normalization. The unity slope obtained by fitting the crossover frequency against DC conductivity data using BNN relation confirms that the $\mathrm{DC}$ and $\mathrm{AC}$ conductions are closely correlated to each other and that they are of the same mechanism.

\section{Acknowledgment}

Author is grateful to Prof. Dr. B. S. Murty, Department of Metallurgical and Materials Engineering, Indian Institute of Technology Madras, Chennai 600 036, India for his support and fruitful discussions.

Received: 21 February 2011 Accepted: 28 February 2012

Published: 9 July 2012

\section{References}

1. Wager JF, Keszler DA, Presley RE: Transparent electronics. Berlin: Springer; 2008.

2. Kawazoe H, Yasukawa M, Hyodo H, Kurita M, Yanagi H, Hosono H: Nature 1997, 389:939.

3. Gao S, Zhao Y, Gau P, Chen N, Xie Y: Nanotechnology 2003, 14:538.

4. Banerjee AN, Chattopadhay KK: J Appl Phys 2005, 97:084308.

5. Prakash T, Padma Prasad K, Ramasamy S, Murty BS: J Nanosci Nanotechnol 2008, 8:4273.

6. Prakash T, Padma Prasad K, Kavitha R, Ramasamy S, Murty BS: J Appl Phys 2007, 102:104104.

7. Namikawa H: J Non-Cryst Solids 1975, 18:173.

8. Chandra Bose A, Balaya P, Thangadurai P, Ramasamy S: J Phys Chem Solids 2003, 64:659.

doi:10.1186/2228-5326-2-12

Cite this article as: Prakash: Influence of temperature on AC conductivity of nanocrystalline $\mathrm{CuAlO}_{2}$. International Nano Letters 2012 2:12 